\title{
Endoscopic Endonasal Resection of a Suprasellar Pituitary Adenoma Mimicking Tuberculum Sellae Meningioma in a Patient with an Intrasellar Persistent Trigeminal Artery
}

\author{
Alaa S. Montaser ${ }^{1,2}$ Alexandre B. Todeschini ${ }^{1}$ Juan M. Revuelta Barbero ${ }^{1}$ Mostafa Shahein ${ }^{1}$ \\ E. Antonio Chiocca ${ }^{3}$ Bradley A. Otto ${ }^{1,4}$ Ricardo L. Carrau ${ }^{1,4}$ Daniel M. Prevedello ${ }^{1,4}$
}

${ }^{1}$ Department of Neurosurgery, Ohio State University Wexner Medical
Center, Columbus, Ohio, United States
${ }^{2}$ Department of Neurosurgery, Ain Shams University Faculty of
Medicine, Cairo, Egypt
${ }^{3}$ Department of Neurosurgery, Brigham and Women's Hospital,
Boston, Massachusetts, United States
${ }^{4}$ Department of Otolaryngology-Head and Neck Surgery, Ohio State
University Wexner Medical Center, Columbus, Ohio, United States

Address for correspondence Daniel M. Prevedello, MD, Department of Neurosurgery, Ohio State University Wexner Medical Center, 410 West, 10th Avenue, Columbus, $\mathrm{OH}$ 43210-1240, United States (e-mail: dprevedello@gmail.com).

J Neurol Surg B 2018;79(suppl S3):S285-S286.

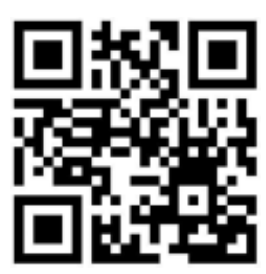

received

October 15, 2017

accepted

December 17, 2017

published online

February 14, 2018
A 50-year-old female with an incidentally diagnosed suprasellar lesion was initially managed conservatively due to the presence of an intrasellar persistent trigeminal artery going through the dorsum sellae and fundamentally forming the blood supply of the entire posterior circulation. Serial follow-up brain magnetic resonance imaging (MRI) revealed progressive enlargement of the suprasellar lesion over 4 years period. Surgery was indicated after the initial tumor growth; however, the patient refused surgery for fear of complications related to the persistent trigeminal artery. Two-and-ahalf years later, she presented with deterioration of vision. Formal visual field testing revealed a right temporal field defect. Brain MRI demonstrated significantly enlarged suprasellar lesion, most consistent with tuberculum sellae meningioma, exerting mass effect on the optic apparatus.

The patient underwent endoscopic endonasal resection of the lesion through a transplanum/transtuberculum approach. Intraoperatively, absence of hypertrophic McConnel arteries, hyperostosis, and the fact that the dura was soft and not under tension was against the diagnosis of tuberculum sellae meningioma. Additionally, the tumor www.thieme.com/skullbasevideos

www.thieme.com/jnlsbvideos

DOI https://doi.org/

10.1055/s-0038-1625943.

ISSN 2193-6331.
๑) 2018 Georg Thieme Verlag KG
Stuttgart · New York

License terms

c) $(1) \$$ 
consistency was similar to a pituitary adenoma. A complete resection was accomplished and multilayer skull base reconstruction was performed with no complications. On postoperative day 1 (POD 1), she was operated upon for the evacuation of small suprasellar hematoma associated with vision deterioration. Histopathological examination confirmed the diagnosis of atypical pituitary adenoma with $K_{i}-67$ labeling index of 4 to $5 \%$. The patient ultimately recovered well with improved vision, and was discharged on POD 4 with no new neurological deficits. At 4 years follow-up, her vision was normalized and brain MRI showed no residual or recurrent lesion.

The link to the video can be found at: https://youtu.be/QZmzctjAEbw.

Conflict of Interest

None.

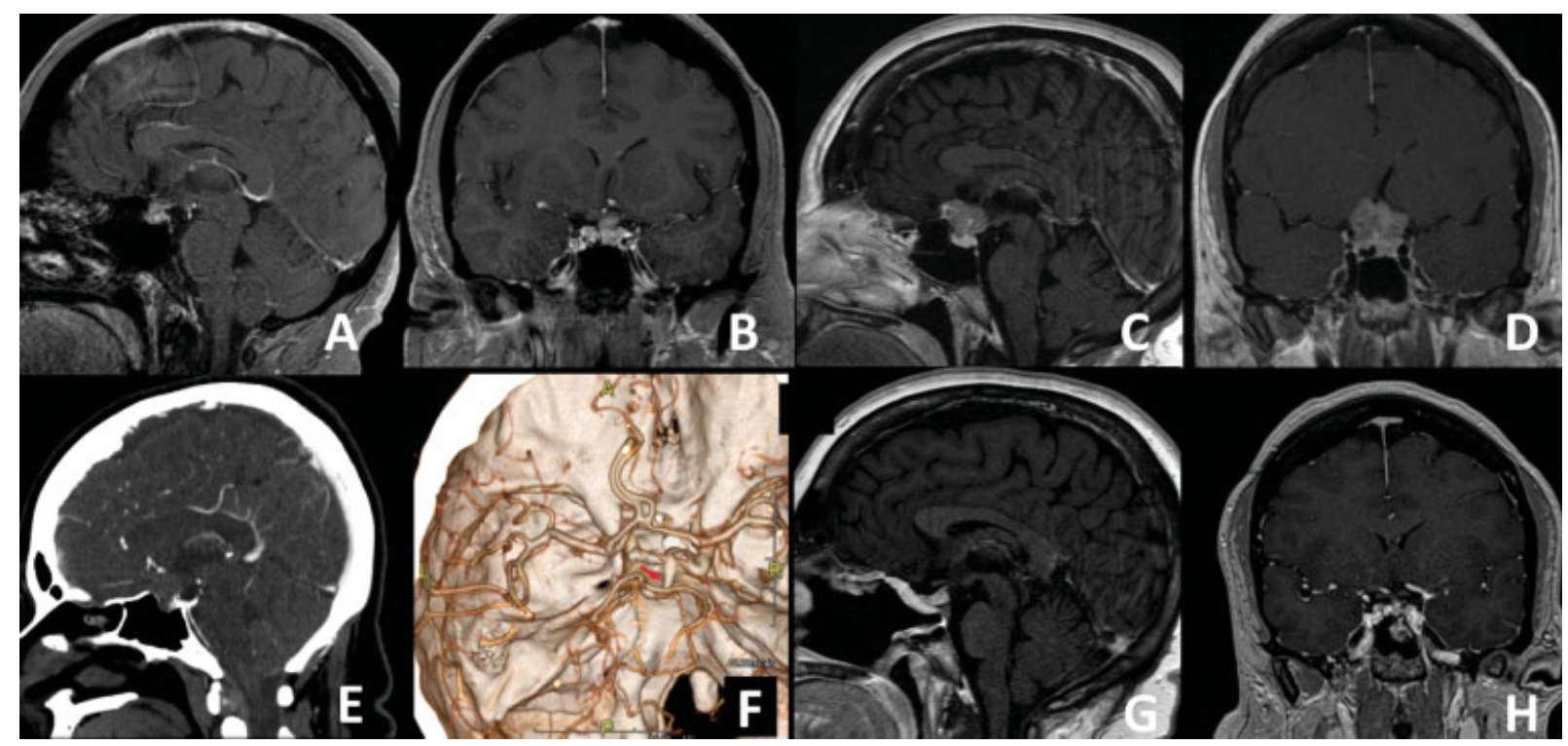

Fig. 1 Initial brain magnetic resonance imaging (MRI) sagittal (A) and coronal (B) views with gadolinium contrast showing the presence of a suprasellar enhanced lesion which was significantly enlarged ( $C$ and $D$ ) over 4 years period. Preoperative computed tomography angiogram sagittal view $(E)$ with three-dimensional reconstruction $(F)$ is showing the persistent trigeminal artery (arrow) tracking through the tuberculum sellae and forming the entire blood supply of the posterior circulation, with absence of the vertebral arteries. Brain MRI sagittal (G) and coronal (H) views with gadolinium contrast performed 4 years after the surgery demonstrating complete resection of the suprasellar adenoma. Note the enhancement of the nasoseptal flap used for skull base reconstruction.

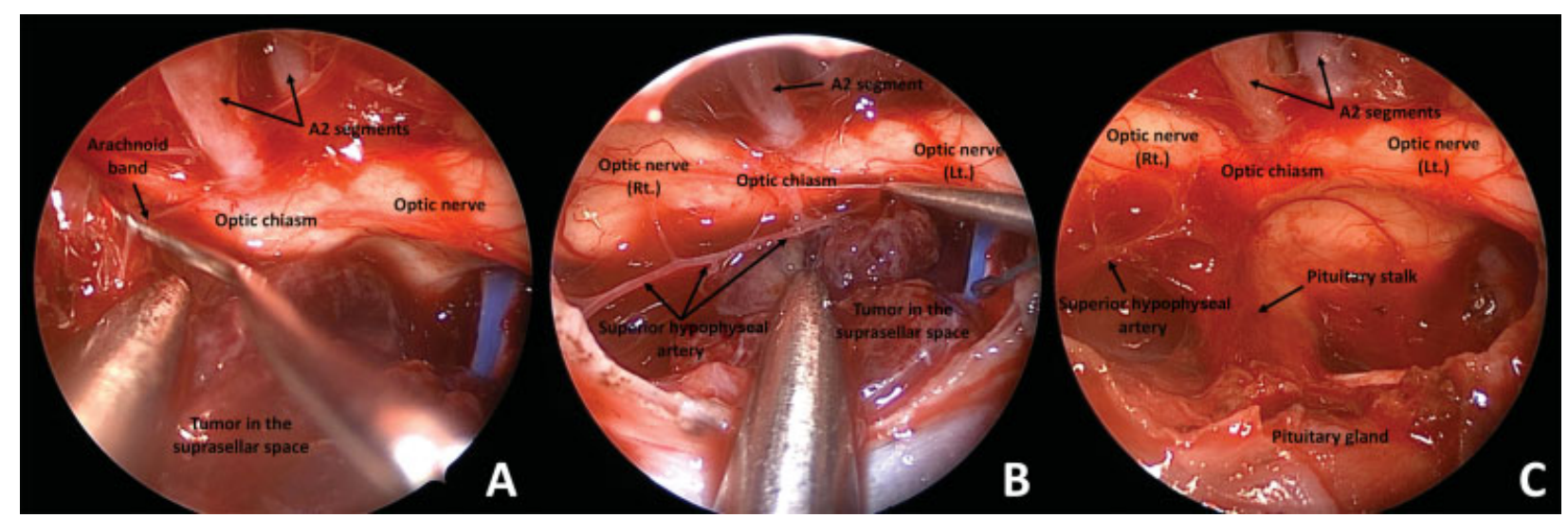

Fig. 2 Intra-op resection of the suprasellar pituitary adenoma. Meticulous dissection of the tumor (A) from the optic chiasm with complete preservation of the superior hypophyseal arteries (B). Complete resection of the tumor (C) was accomplished. 